\title{
Is personalized medicine a panacea for health management? Some thoughts on its desirability
}

\author{
Fernando Antoñanzas • Carmelo A. Juárez-Castelló • \\ Roberto Rodríguez-Ibeas
}

Published online: 12 December 2014

(c) Springer-Verlag Berlin Heidelberg 2014

During the last decade we have witnessed a change in health care management. Predictive, personalized [1], individualized [2] or stratified [3] medicine are some of the terms that have been coined to describe a new approach to dealing with disease. In essence, the new paradigm is based on matching patients with the best treatments available according to each patient's characteristics. Patients are classified through new tests, mainly genetic in nature, the growing proliferation of which is behind the extensive application of this new approach to managing disease. In parallel, new therapies, many based on new mechanisms of action and biological agents, allow ever narrower targets within the cell to be addressed. This combination of new diagnostic techniques and targeted therapies has improved the perspectives of managing some difficult conditions, such as some types of cancer, by controlling the progression of a disease and maintaining it rather as a chronic condition, even achieving a cure in some cases. This new paradigm is raising expectations for both patients and physicians. Health care systems are transforming the management of some diseases and re-writing treatment guidelines to incorporate the advantages of newly available therapies and approaches. However, notwithstanding the rapid development of these changes, there are some issues that should also be taken into account. Here, we highlight some elements that are frequently overlooked or taken for granted.

Personalized medicine is frequently based on drug treatments that are more expensive than other, more traditional, therapies. These types of new drug generally target conditions that are more prevalent in developed

F. Antoñanzas $(\varangle)$ · C. A. Juárez-Castelló · R. Rodríguez-Ibeas University of La Rioja, Logrono, Spain

e-mail: fernando.antonanzas@unirioja.es countries, where a wide market in terms of potential patients and purchase capacity exists. To our knowledge, there is no experience of this kind of approach being used to address conditions whose prevalence is higher in less developed countries. It would be interesting to make personalized medicine accessible to lower income countries so that they could also benefit from the new advantages.

A clear contribution of personalized medicine is that it aims to address the imperfect information related to diagnoses and effectiveness that generates uncertainties in healthcare. Personalized medicine reduces uncertainty by identifying more clearly those patients that will response better to treatment and by establishing new and more specific therapeutic targets. However, personalized medicine could also have the "side effect" of introducing undesirable effects into the system, in the sense that new diseases are seemingly created based on an identified gene mutation. Manufacturers intend to classify mutations of the general condition as potential new orphan diseases (prevalence lower than 50 cases per 100,000 persons), which, as a consequence, would attract a greater reward from health authorities in terms of higher prices for the new therapy, and probably better reimbursement policies. Of course, this process would imply higher costs for public health care systems. This is nothing new at this point, however, as this trade-off between improved health outcomes and higher health costs is already common practice.

Within the last decade, new tests, mostly genetic, have gained access to the market. Currently, there are over 1,800 genetic tests that help define and classify new diseases and patients [4]. Some tests belong to the field of so-called companion tests, i.e., they must be applied to patients prior to the administration of a given drug to make sure that they are the right candidate to receive that medication. Other tests do not belong to this category and their link to drug 
treatment is not as narrow. In either case, the pressure to increase the number of diagnostic procedures keeps growing and the effects, generally on hospital budgets, are becoming a concern. Consider the case of a low prevalence condition for which a genetic test to identify better responders to a given treatment is available. The management of that disease would advise prior administration of the test to any patient suspected of having the disease. This would necessitate that, besides the personalized treatment costs of the best responders, the costs of the test be applied to the whole population of patients. Depending on the costs of the tests and therapies, this way of coping with the disease may incur excessively high costs, making the application of personalized medicine unadvisable [5].

The economic literature is not fully conclusive on whether personalized medicine leads to either global savings or cost increases in health budgets. Clearly, there are some areas where the combination of tests and personalized therapies will generate savings in the management of the disease (e.g., the KRAS test in colorectal cancer saves costs and improves health outcomes). However, in other areas, the application of personalized medicine will definitely increase health costs, although simultaneously contributing to health improvements. In these cases, to adopt efficiently guided decisions, it will be necessary to develop economic evaluations adapted to each particular case.

Regarding the application of economic evaluation to personalized medicine, the development of such analysis is far from straightforward. The specific features of evaluation processes (mainly assessing the links between tests results and final health outcomes) demand ever more complex techniques to assess the final effectiveness of the technologies under study as well as their efficiency [2]. Currently, much research is being undertaken in this area, as can be seen in the literature and in ISPOR discussion panels.

To date, genetic tests, including the reagent kits required to implement them, have not received the attention they should given their consequences for the therapeutic decisions derived from their results. The quality of a test depends on its sensitivity and specificity to classify patients together with its inherent characteristics (ease of application both to patients and in the laboratory where the results are obtained) [5]. The originator of a diagnostic test guarantees its value by mean of the CE mark; interestingly, the CE mark is always a self-assessed certification. Thus, it would seem that more effort and resources should be devoted to externally validate the quality of such tests, thereby reducing some inherent uncertainties and asymmetries of information.

The CE mark has become a requirement for market access in the European Union (EU). This mark sends a positive signal of quality to the market, allowing manufacturers to market the new test more profitably. Furthermore, hospital laboratories have also developed their "own cuisine" tests, that is to say, they use some of the commercial reagents, assays and kits to combine them in their own way to diagnose diseases. In these cases, assessment of the quality of the final result can even be more subject to uncertainties and wider variability and, again, some external assessment would be desirable.

In some cases, industrial laboratories develop a test together with a new drug suitable for a group of patients. These tests, when marketed jointly with the drug, are called companion tests. Previously, a clinical trial must have demonstrated that patients can be identified by the test and that the treatment is adequate for them. This process may require a period longer than usual to jointly assess the value of the innovation. Then, having approved the evidence, in the case of the EU, the European Medical Agency (EMA) would bind together the test and the drug and authorize the joint new technologies.

Nevertheless, for diseases with limited therapeutic options, early access mechanisms have been applied. A case in point is the recent market approval by the EMA in 2014 of Vynfinit ${ }^{\circledR}$, together with the two diagnostic agents Folcepri ${ }^{\circledR}$ and Neocepri ${ }^{\circledR}$ (all of them are designated orphan medicines), targeting platinum-resistant ovarian cancer. According to the EMA's own explanation, this approval "illustrates the current trend towards the development of medicines targeted at specific patient populations, which is based on a better understanding of the underlying molecular mechanisms of the disease. By targeting specific patient groups who are likely to better respond to a treatment, the response rate in the population treated can be improved and treatment can be avoided in patients who are unlikely to respond to the treatment, sparing them potential side effects" [6].

We must point out that national processes for price and reimbursement authorization are more complex as health regulatory bodies must assess not only the efficacy of the drug - as was traditionally the case--but also the discriminatory power (i.e., the classification of potential patients to receive the drug) of the test as a prior step in order to understand the final health outcomes. The test plus medication bundle must be compared to the standard of care without any previous stratification of patients, which makes the assessment process more cumbersome. It has also been acknowledged that such joint authorization will give the manufacturer monopoly power as no other alternative test can be used for the identification of patients suffering from the condition prior to treatment with the new agent.

The rapid development of new genetic tests illustrates the possibilities opened by genomics to better understand human biology and to identify new therapeutic targets. 
However, we must be aware of the risks of having too many slightly different tests with small therapeutic value. On the one hand, in general terms, it is good in any emerging area to have a lot of different sources of information; however, on the other hand this abundance of tests may also be perceived as a challenging strategy used by manufactures to artificially differentiate themselves-both in terms of the tests and in terms of new therapies- to increase their market power and profits.

In conclusion, we observe that current medical management of disease processes is based on several diagnostic procedures, including some that are genetic in nature, which allow a therapeutic plan to be established. Due to the inherent uncertainty surrounding the validity of test results (false positives and false negatives) and treatment effectiveness, it still remains ambiguous if personalized medicine is the best choice for all conditions [2]. For instance, there are some reported cases of patients responding to oncology treatments in spite of being previously classified by genetic tests as not indicated to receive that particular therapy. This fact leads to some doctors having consistent doubts on how to manage some patients. Furthermore, as this new approach implies additional expenses in many cases, the change in paradigm from traditional management of disease to personalized medicine should be based not only on potential health gains but also on information derived from an economic analysis of these new technologies. Personalized medicine is not yet a panacea; in our view, its implementation demands case-by-case interdisciplinary assessment.

\section{References}

1. Rogowski, W., Payne, K., Schnell-Inderst, P., Manca, A., Rochau, U., Jahn, B., Alagoz, O., Leidl, R., Siebert, U.: Concepts of "Personalization" in personalized medicine: implications for economic evaluation. PharmacoEconomics (2014). doi:10.1007/ s40273-014-0211-5

2. Hatz, M.H.M., Schremser, K., Rogowski, W.H.: Is individualized medicine more cost-effective? A systematic review. Pharmaco. Economics 32, 443-455 (2014)

3. Trusheim, M.R., Berndt, E.R., Douglas, F.L.: Stratified medicine: strategic and economic implications of combining drugs and clinical biomarkers. Nat Rev Drug Discov 6, 28793 (2007)

4. Antoñanzas, F., Rodríguez-Ibeas, R., Hutter, M.F., Lorente, R., Juárez, C., Pinillos, M.: Genetic testing in the European Union: does economic evaluation matter? Eur J Health Econ 12(5), 651-662 (2012)

5. Antoñanzas, F., Juárez-Castelló, C.A., Rodríguez-Ibeas, R.: Some economics on personalized and predictive medicine. Eur J Health Econ (2014). doi:10.1007/s10198-014-0647-8

6. European Medicines Agency (EMA): EMA recommends approval of new treatment for platinum-resistant ovarian cancer together with companion diagnostic. Available at http://www.ema.europa. eu/ema/index.jsp?curl=pages/news_and_events/news/2014/03/ news_detail_002051.jsp\&mid=WC0b01ac058004d5c1, accessed 28 November 2014 